Service social

L'intervention collective en travail social : l'action auprès des groupes et des communautés, par Christina De Robertis et Henri Pascal, Paris, Éditions du Centurion (Socioguides) 1987, 303 pages.

\title{
Ginette Berteau
}

Volume 38, numéro 1, 1989

Aspects psychosociaux du Sida

URI : https://id.erudit.org/iderudit/706429ar

DOI : https://doi.org/10.7202/706429ar

Aller au sommaire du numéro

Éditeur(s)

École de service social de l'Université Laval

ISSN

1708-1734 (numérique)

Découvrir la revue

Citer ce compte rendu

Berteau, G. (1989). Compte rendu de [L'intervention collective en travail social :

l'action auprès des groupes et des communautés, par Christina De Robertis et

Henri Pascal, Paris, Éditions du Centurion (Socioguides) 1987, 303 pages.]

Service social, 38(1), 133-136. https://doi.org/10.7202/706429ar d'utilisation que vous pouvez consulter en ligne.

https://apropos.erudit.org/fr/usagers/politique-dutilisation/ 


\section{RECENSIONS}

L'intervention collective en travail social: l'action auprès des groupes et des communautés, par Christina De Robertis et Henri PASCAL, Paris, Éditions du Centurion (Socioguides) 1987, 303 pages.

Ce livre est, selon nous, un ouvrage de sensibilisation destiné à des travailleurs sociaux qui, d'abord formés à l'intervention individuelle, aimeraient se familiariser avec les différents concepts, outils d'analyse et d'intervention requis par le travail auprès des groupes et des unités sociales restreintes (quartier, village, institution résidentielle).

En introduction, les auteurs nous informent que leur ouvrage réfère aux mêmes concepts de base et utilise le même découpage des phases de la méthode d'intervention sociale qu'une première parution : "Méthodologie de l'intervention en travail social» (1984). Ils désirent mettre l'accent sur les aspects unificateurs des diverses méthodes d'intervention sociale plutôt que sur leurs différences.

Le volume comporte trois grandes parties :

- L'intervention collective : définition et méthode

- Les outils d'analyse

— Les outils d'intervention

\section{L'intervention collective : définition et méthode}

Cette première partie (chapitres 1 et 2 ) situe d'abord historiquement la place occupée par le service social de groupe et l'intervention collective dans la formation et la pratique des travailleurs sociaux. On y apprend que l'intervention individuelle est la méthode la plus utilisée en France et que, présentement, les travailleurs sociaux français redécouvrent les autres méthodes d'intervention. Les auteurs mettent alors en relief l'influence de la conception du modèle médical sur la pratique des travailleurs sociaux.

Vient ensuite la définition des termes: développement communautaire, organisation communautaire et service social de groupe. Pour exposer ces concepts, les auteurs s'appuient sur de solides références de base (Ross, Paré); on aurait cependant espéré qu'ils puisent à des sources plus récentes.

Dans leur définition du service social de groupe, De Robertis et Pascal s'inspirent de la typologie d'Hélène Massa (1976) établissant deux modes d'intervention en groupe : le traitement social et les modèles à buts sociaux. Ils ne font aucune mention du modèle de réciprocité (basé sur l'aide mutuelle) sinon de souligner, au chapitre sur les outils d'analyse, qu'un groupe peut s'entraider. 
Sans dénigrer la typologie utilisée, il nous semble que les auteurs auraient pu introduire la notion d'utilisation de l'aide mutuelle, cette dernière étant une des principales caractéristiques du service social de groupe.

Malgré les distinctions apportées entre les divers concepts, le livre est ambigu en ce qui concerne la définition de l'intervention collective. Tout ce qui n'est pas une intervention individuelle semble être une intervention collective. Ainsi, les auteurs décrivent une intervention auprès d'un groupe d'enfants ayant des difficultés à établir des relations harmonieuses. Le but du groupe est d'utiliser des supports relationnels autres que la parole. De Robertis et Pascal associent ce type d'action à une intervention collective. Selon notre cadre de référence, cette situation ne relève pas de l'intervention collective (changements de structures de la société, focalisation sur des problèmes collectifs) mais plutôt d'une intervention de groupe axée sur le changement personnel.

Un peu plus loin, les auteurs décrivent les phases de la méthode d'intervention collective que nous dénommons ici le processus rationnel d'intervention.

\section{Les outils d'analyse}

Cette partie traite des outils permettant de comprendre une réalité sociale. Le chapitre 3, consacré à l'enquête sociologique, met l'accent sur la contribution de la recherche à une meilleure compréhension des situations-problèmes et à une plus juste analyse du processus et des résultats de l'intervention. Les auteurs donnent un aperçu général des règles méthodologiques d'une démarche scientifique en recherche; ils insistent particulièrement sur les techniques d'enquête telles que : techniques d'observation, d'entretien, de comptage, et techniques expérimentales. Ce chapitre se termine sur une vision globale des champs de recherche en service social.

Le chapitre 4 enchaîne avec la description détaillée d'un deuxième outil d'analyse: l'étude de milieu ou l'étude du contexte global en fonction des besoins manifestés. L'utilisation de cet outil permet au travail social de prendre une distance par rapport à la situation présentée et surtout de se donner une vue d'ensemble. Les auteurs indiquent également comment faire une étude de milieu.

Le troisième outil est l'analyse des organisations (chapitre 5). L'interrelation entre les organismes distributeurs de services et les problématiques sociales est mise en évidence. Pour les auteurs, les organisations sont des déterminants-clés dans la lecture que les travailleurs font des situations.

Le dernier outil est l'analyse des groupes (chapitre 6). Les auteurs insistent pour qu'un groupe soit analysé en fonction du contexte social dans lequel il évolue. Une grille dégage à la fois des indicateurs sur les phénomènes internes au groupe (processus de communication - d'interaction - influence de prise de décision) et sur ceux qui lui sont externes (appartenance à d'autres groupes et relations inter-groupes).

Cette section sur les outils d'analyse nous apparaît pertinente puisqu'elle démontre nettement l'importance d'utiliser des instruments tels que la démarche 
scientifique, l'étude de milieu, l'analyse des organisations et des groupes lors de l'élaboration d'un projet d'intervention collective. Les grilles proposées peuvent être des points de repère intéressants pour les intervenants voulant s'initier à ce type d'approche. Cependant, dans leur souci de passer en revue tous les outils possibles d'analyse, les auteurs développent peu les particularités de chacun; par conséquent, l'intervenant voulant utiliser tel instrument devra se référer à d'autres sources.

\section{Les outils d'intervention}

De Robertis et Pascal voient les outils d'intervention comme les moyens d'action à privilégier lors d'une intervention collective. Ces moyens sont développés à l'intérieur de quatre chapitres : création de structures d'intervention (chap. 7); intervention auprès des groupes (chap. 8) ; interventions spécifiques aux grands groupes (chap. 9); intervention auprès d'une population (chap. 10).

À l'intérieur du chapitre destiné à la création de structures d'intervention, les auteurs mettent d'abord de l'avant des moyens de collectiviser les situations, à partir de demandes ou besoins individuels. Puis, ils élaborent sur les étapes chronologiques de la mise sur pied d'un groupe initié par un travailleur social. Ils insistent particulièrement sur l'évaluation des besoins en indiquant les impacts sur les résultats de l'intervention. Finalement, ce chapitre dégage les particularités des outils d'intervention auprès d'un groupe formé de participants provenant de diverses instances ou organisations.

Le chapitre suivant décrit l'intervention directe auprès d'un groupe. Les auteurs revoient ici les principales tâches d'un travailleur social à chacune des phases d'une rencontre : démarrage, productivité (équilibre entre la tâche, la vie socio-affective et le développement de l'entraide) et fin. Une attention particulière est portée à la notion de programme d'activités ; les auteurs précisent qu'un tel programme doit être élaboré en fonction des besoins des membres et en démontrent l'utilité pour le développement du groupe. Ils terminent en insistant sur l'importance de l'évaluation continue en intervention de groupe.

Au chapitre suivant, les auteurs précisent les caractéristiques des grands groupes et abordent les moyens d'organiser des rencontres avec ceux-ci : conférence, panel, tribune ouverte, table ronde, débat, Philipps 66, etc.

Le dernier chapitre de cette section est consacré au travail auprès d'une population. On y précise les objectifs à atteindre ainsi que les techniques à utiliser : enquête conscientisante, techniques d'information et d'animation. Les auteurs terminent ce chapitre en discutant des stratégies d'intervention à développer lors d'une action auprès d'une population.

Cette section sur les outils d'intervention représente le savoir-faire de l'ouvrage. Elle a le mérite de regrouper dans un même document les tâches et les techniques les plus courantes utilisées auprès des petits et grands groupes et auprès d'une population.

Notons aussi qu'une des grandes forces de l'ouvrage est de faire ressortir les liens entre les diverses méthodes d'intervention sociale, leurs outils d'analyse et 
les moyens d'application. Les paramètres les plus importants de la pratique en service social y sont présents : définition des concepts, recherche, processus rationnel d'intervention, évaluation. Les auteurs parviennent à nous faire voir comment ces divers éléments sont essentiels au choix d'une intervention appropriée et à sa réussite.

Cela fait un livre à la fois riche et lourd. Riche étant donné l'aspect unificateur des principaux paramètres de la pratique, lourd parce que tout est traité de façon générale, rien n'est analysé ni décrit en profondeur.

Certaines parties de l'ouvrage nous apparaissent mieux menées, entre autres la section sur les outils d'analyse et d'intervention. La partie la plus décevante est, sans contredit, le chapitre portant sur les définitions et les concepts: on y souhaiterait des références plus récentes de même qu'une cohérence entre définitions et exemples.

De par son approche globale et synthétique, cet ouvrage peut être d'un usage pertinent pour tout intervenant qui veut se sensibiliser aux méthodes destinées aux petits groupes et aux collectivités. Il peut aussi apporter un certain éclairage aux étudiants en service social en leur permettant de faire des liens entre les thèmes les plus importants de leur formation.

Bref, voilà un bel effort de systématisation des différents aspects de la pratique autre qu'individuelle. Cependant, cet ouvrage a des forces (savoirfaire) et des limites (niveau conceptuel). Mais peut-être sommes-nous mauvais juge ; nous présentons ce livre à la lumière de notre réalité québécoise et nordaméricaine, alors qu'il a probablement une toute autre portée en Europe.

Ginette BerTeAu

Chargée de cours en intervention de groupe,

Université Laval et Université de Montréal.

Les homosexuels et le sida: sociologie d'une épidémie, par Michael Pollak, publié avec le concours de la Mission Recherche Expérimentation, Paris, Éditions A.M. Métailié, 1988, 220 pages.

Michael Pollak, chercheur au Conseil National de Recherche Sociale (CNRS) à Paris, offre ici une synthèse de ses travaux présentés aux conférences internationales sur le sida. Dès la présentation, l'auteur ouvre l'angle de la réflexion et même du débat qu'il soutiendra tout au long de l'ouvrage : "Comment faire passer le message préventif [concernant le sida] sans provoquer de dramatisation excessive qui alimenterait des réactions répressives médicalement injustifiées?" (p. 14).

La première partie est consacrée à une meilleure connaissance de la communauté homosexuelle française en regard de la problématique du sida. Elle se fonde sur des comptes rendus de recherches quantitatives et qualitatives, soit : 